\title{
New chapter in tackling antimicrobial resistance in Thailand
}

In figure 1 of this article (BMJ 2017;358:j3415, doi:10.1136/ bmj.j2423) the goal for reduction in animal use of antimicrobials was incorrectly stated as $20 \%$ instead of $30 \%$. The article has been updated to correct the figure. 